\title{
Microwave Surface-Plasmon-Like Modes on Thin Metamaterials
}

\author{
Matthew J. Lockyear, Alastair P. Hibbins, and J. Roy Sambles \\ School of Physics, University of Exeter, Stocker Road, Exeter EX4 4QL, United Kingdom
}

(Received 11 November 2008; published 17 February 2009)

\begin{abstract}
It has recently been shown that the structured surface of a perfect conductor can support surfaceplasmon-like modes [Pendry et al., Science 305, 847 (2004)]. Such structures have a thickness of at least the order of the wavelength. Here, using microwave wavelength radiation incident beyond the critical angle of a wax prism, we quantify the surface-plasmon-like dispersion for a metamaterial surface with a thickness very much smaller than the incident wavelength.
\end{abstract}

DOI: 10.1103/PhysRevLett.102.073901

PACS numbers: $42.25 . \mathrm{Bs}$

The guiding of electromagnetic (EM) surface waves at the planar interface between two dissimilar media has been the subject of much theoretical and experimental study since the early twentieth century $[1,2]$. In the visible domain the surface waves supported are known as "surface plasmons" [3]. They are TM polarized and propagate just below the speed of light, being cutoff at the surface plasma frequency $\left(\omega_{p} / \sqrt{1+\varepsilon_{0}}\right.$, where $\omega_{p}$ is the plasma frequency of the substrate). Close to this limiting frequency (typically in the ultraviolet), the mode becomes tightly bound to the interface with enhanced fields on resonance that decay exponentially into the media either side. However, for much lower frequencies, including the microwave spectrum, the dielectric constants of metals become large and imaginary causing such surface waves to propagate almost without attenuation at speeds close to the speed of light. In this regime, these loosely bound modes are often described as surface currents. However, as early as 1944, researchers were aware that the addition of a subwavelength corrugation to the metal surface would produce an enhanced surface reactance and strongly bind the mode to the interface, even in this long-wavelength regime [[4-6] and references therein].

One simple form of corrugation is an array of closely spaced, narrow vertical slots in a metal substrate. If the slots are resonant, e.g., approximately one-quarter of a wavelength deep, then the short circuit (their closed end) at the bottom is transformed by the length of the slot into an open circuit at the open end, producing a high impedance surface (HIS). For wavelengths longer than 4 times the slot depth, the effective surface impedance is positive and imaginary and enhanced above that of a planar metal. Such surfaces are thereby able to support TM-polarized bound surface waves which are cut off at the resonant frequency, analogous to the limiting surface plasma frequency of metals in the visible regime. A similar discussion was recently presented by Pendry et al. [7]. They considered an array of subwavelength holes in a thick metal substrate and analytically derived the dispersion of the surface wave supported. The current authors later experimentally verified their proposal, using finite depth holes, confirming that the dispersion of these "spoof" surface plasmons are asymptotic to the resonance of the lowest energy waveguide resonance of the void. Since then, several interesting plasmonic surfaces have been reported upon in both the microwave and $\mathrm{THz}$ regime [8-11], notably concerning the confinement of surfaceplasmon-like modes and the propagation of these modes on corrugated metal wires.

Another version of the HIS that for lower frequencies may support spoof surface plasmons is the "Sievenpiper mushroom" structure [12]. Here the quarter wavelength slots have been embodied in to a subwavelength structure that possesses both capacitive and inductive components. Its resonant properties can be explained using a simple circuit model (Fig. 1. inset), where the surface impedance is given by $Z_{s}=i \omega L /\left(1-\omega^{2} L C\right)$. In a similar manner to the slotted surfaces discussed, this surface is inductive below the resonant (high impedance) frequency $\omega_{0}=$ $(L C)^{-1 / 2}$, and may support a bound TM surface wave. This provides a surface which supports the localization of a surface wave but on a structure whose thickness is only a fraction of the wavelength $(<1 \mathrm{~mm})$, compared to the much thicker "Pendry" and corrugated slab structures. In this study, we use detailed experimental analysis and finite element modelling [13], to illustrate strong coupling of incident microwave radiation to a bound TM surface mode on such a structure.

The surface studied here [Figs. 1(a) and 1(b)] is comprised of square $18 \mu \mathrm{m}$ thick copper patches of side length $a=1.3 \mathrm{~mm}$ arranged in a square array of pitch $\lambda_{g}=$ $1.6 \mathrm{~mm}$. The patches are separated from a copper ground plane by a low loss $0.79 \mathrm{~mm}$ thick dielectric layer [Nelco NY9220 $(\varepsilon=2.22+0.002 i)]$; however, each individual copper patch is connected to the ground plane by a hollow copper via of radius $r_{v}=0.15 \mathrm{~mm}$. Since the unit cell is subwavelength $\left(\lambda_{g}=1.6 \mathrm{~mm}\right.$ compared to an incident wavelength range of $10<\lambda_{0}<30 \mathrm{~mm}$ ) the surface may be represented by lumped circuit theory as a capacitor and inductor connected in series [Fig. 1(b), inset]. Although this simple model does not predict perturbation of the surface mode adequately since the band gap by definition 


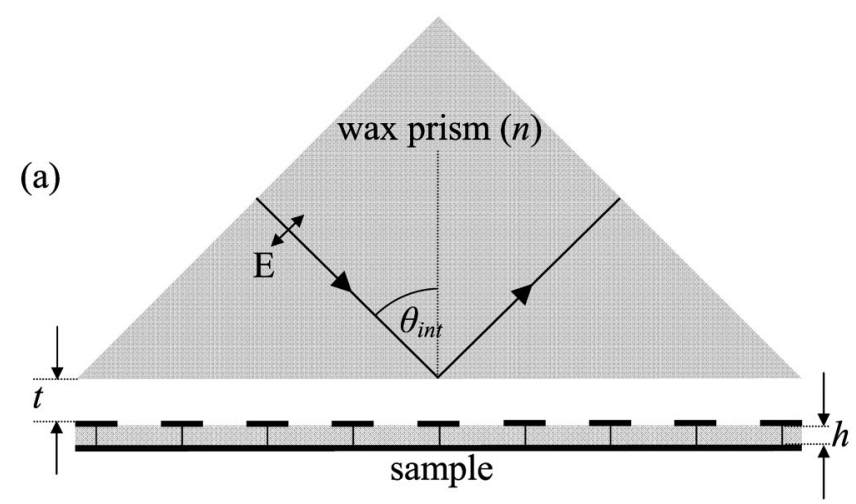

(b)

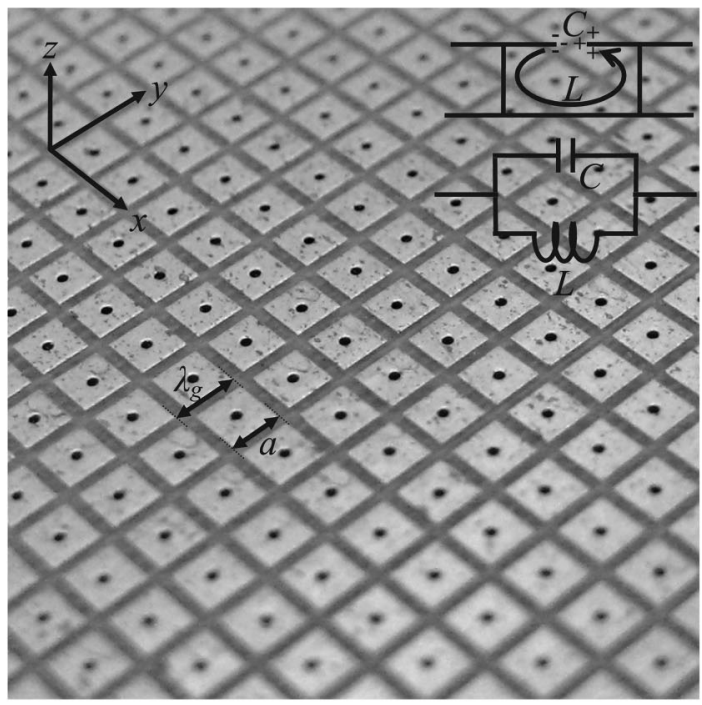

FIG. 1. (a) The sample, of core thickness $h=0.79 \mathrm{~mm}$. With microwave radiation incident beyond the critical angle of the prism, the spacing $t$ provides a suitable tunnel barrier to allow the evanescent fields from the prism to couple to the TM surface mode supported by the structure in the standard Otto configuration. (b) Each individual patch is connected to the ground plane by a hollow copper via. Inset: a unit cell of the array may be described to first order by lumped circuit theory, the capacitive contribution to the resonance arising from capacitive coupling between nearest neighbor patches, and the inductive component from oscillating currents between nearest neighbors.

extends to high wave vectors, where the wavelength becomes smaller than the individual features of the structure described, it does predict the EM response of the sample to first order, with the capacitive component of the resonance arising from the capacitive coupling between nearestneighbor patches, and the inductive contribution from oscillating surface currents between adjacent unit cells [see Fig. 1(b), inset].

Collimated plane wave radiation is incident via a large wax prism $(n=1.5)$ at an angle beyond the critical angle $\left(\theta_{\text {int }}>\sin ^{-1} 1 / n\right)$. By spacing the prism above the sample by a distance $t$ to provide a suitable tunnel barrier, the evanescent fields beyond the prism resonantly couple to the TM mode supported by the surface (Otto geometry [14]).
Reflected radiation from the sample is collected using a spherical mirror focused to a detector, allowing the resonance to be observed as a strong reflectivity minimum in the specularly reflected beam, due to power being dissipated on resonance into the dielectric core, and via Joule heating of the metal. For a range of incident angles $\left(42^{\circ}<\right.$ $\theta_{\text {int }}<71^{\circ}$ ), the parameter $t$ is varied, allowing the optimum coupling condition to be found. For this optimum condition, the resonant frequency of the mode was evaluated allowing the in-plane wave vector $k_{\|}$to be extracted and the mode's dispersion constructed.

The inset in Fig. 2 shows a typical reflectivity spectrum from the sample as a function of tunneling gap $t$. In this instance, plane wave radiation $\left(18<f_{0}<26 \mathrm{GHz}\right)$ is incident at an internal angle of $58^{\circ}$, with the incident electric vector confined to the plane of incidence (TM polarization). It is clear that variation of the parameter $t$ not only brings the resonant coupling through an optimum condition $\left(t=3.5\right.$ through $7.5 \mathrm{~mm}$ with $\left.t_{\mathrm{opt}}=5.5 \mathrm{~mm}\right)$, but also shifts the resonant frequency of the mode. This shift is predominantly due to the exponentially decaying fields associated with the TM surface mode significantly penetrating the wax prism. Ideally, $t$ would be increased to a limiting frequency (at which the resonant frequency of the mode ceases to change with an increase in tunneling gap), ensuring that the penetration of the fields into the prism no longer perturb the modes dispersion. However, for the

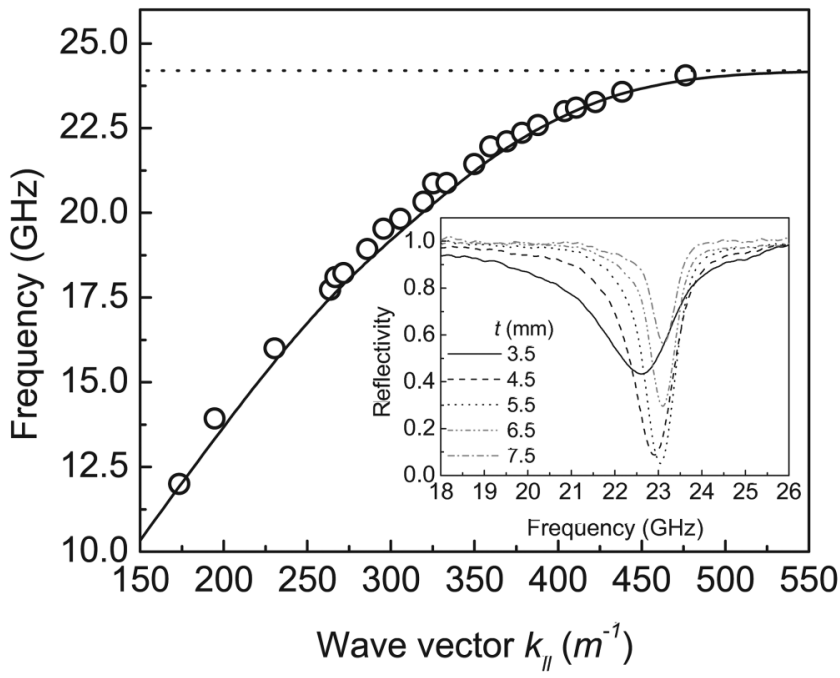

FIG. 2. (circles) The dispersion of the TM surface mode supported by the sample compared to the predictions of the finite element method model (solid line). The frequency of the lower band edge associated with the sample is also shown (dotted line), to which the dispersion is asymptotic. Inset: Specular reflection from the sample as a function of frequency for $\theta_{\text {int }}=58.2^{\circ}$. Here, the tunnel gap $t$ is varied between 3.5 and $7.5 \mathrm{~mm}$ in $1 \mathrm{~mm}$ steps. The resulting shift in frequency is attributed to field penetration into the wax prism, due to its close proximity to the surface. Note an optimum coupling condition at $t_{\mathrm{opt}}=$ $5.5 \mathrm{~mm}$. 
majority of angles as this limit is approached, coupling becomes too weak and the mode becomes too broad for accurate experimental determination of the resonant frequency. Instead we limit ourselves to recording the resonant frequency at the optimum coupling condition for all angles.

Figure 2 (circles) shows the dispersion of the TM surface mode at the optimum coupling condition for a range of internal angles between $42^{\circ}<\theta_{\text {int }}<71^{\circ}$. Also shown (solid line) are the predictions from the finite element method model. Here, as in the experimental case, the mode dispersion is calculated at the optimum coupling condition, by constructing a unit cell of the sample and making use of EM symmetry planes to approximate to an infinite plane wave impinging on an infinite sample area. TM-polarized radiation is incident through a medium simulating the wax prism, and a suitable tunnel barrier is provided by a vacuum layer of variable thickness. The experimental and theoretical results compare extremely well considering the experimental arrangement inherent beam spread $\left( \pm 1^{\circ}\right)$, which is not taken into account by the model. We also approximate to an infinite plane wave impinging on an infinite sample area.

The fundamental resonant frequency $f_{\text {res }}$ for this sample may be calculated from its geometrical parameters using an effective surface impedance model [15]. In the limit $\lambda_{g}<\lambda_{0}$, and for normal incidence, an expression for $Z_{s}$ may be obtained:

$$
Z_{s}=\frac{j \frac{\eta}{\sqrt{\varepsilon_{r}}} \tan \left(k^{\prime} h\right)}{1-\frac{\left(\varepsilon_{r}+1\right) k D}{\pi \sqrt{\varepsilon_{r}}} \log \left(\frac{2 \lambda_{g}}{\pi w}\right) \tan \left(k^{\prime} h\right)} .
$$

Here $w$ represents the distance between nearest neighbor patches $\left(\lambda_{g}-a\right), \varepsilon_{r}$ and $h$ are the dielectric constant and thickness of the dielectric core, respectively, $\eta$ is the free space impedance and $k^{\prime}=k / \sqrt{\varepsilon_{r}}$, where $k$ is the free space wave vector. Plotting $Z_{s}$ as a function of $k$ allows the resonant frequency of the structure to be obtained by observing the singularity in (1) at $f_{\text {res }}$. Numerically solving this equation predicts $f_{\text {res }}=33.4 \mathrm{GHz}$ for the given sample parameters. It is clear however that the mode dispersion approaches an asymptotic limit somewhat lower than $f_{\text {res }}$. This is as expected due to the perturbation of the mode dispersion by the band gap (the finite element method model takes into account the Brillouin zone boundary at $k_{g} / 2$ whereas the much simplified lumped circuit theory does not). As previously discussed, it is $Z_{s}$ that defines the boundary conditions for the surface mode. Using the effective surface impedance model described in (1), the reflection phase for the sample studied here can be used to define the position in frequency of the band gap [16], with the lower edge of the band $\omega^{\prime}$ being approximated by the expression

$$
\omega^{\prime 2}=\omega_{0}^{2}\left(1-\frac{Z_{s}}{\eta}\right)
$$

If we assume that the bandwidth is symmetric about $f_{\text {res }}$, this expression yields a lower band edge frequency for this sample of $24.2 \mathrm{GHz}$ (dotted line of Fig. 3).

Although it is clear from Fig. 2 that the dispersion of the TM surface mode is asymptotic to the effective surface plasma frequency of the sample and therefore has the required surface plasmon form, for this mode to be considered a true surface wave, strong power flow must occur across the surface of the array. In addition, to make a connection to the plasmon like modes discussed in [7], we also expect exponentially decaying fields into at least the air on resonance. It is therefore useful to evaluate the fields at a frequency corresponding to the resonant frequency of the TM mode using the finite element method model.

Figure 3(a) (inset) shows the time averaged electric field magnitude (gray scale) evaluated over a surface in the $y z$ plane (plane of incidence) through the center of a unit cell of the array (through the center of a connecting via). In this instance, $\theta_{\text {int }}=51.2^{\circ}$. The lightest regions correspond to areas with largest field enhancements (white corresponds to a field enhancement of 40 times the incident field). It is clear that the region of highest field enhancement is confined to be within only a few millimeters of the surface of

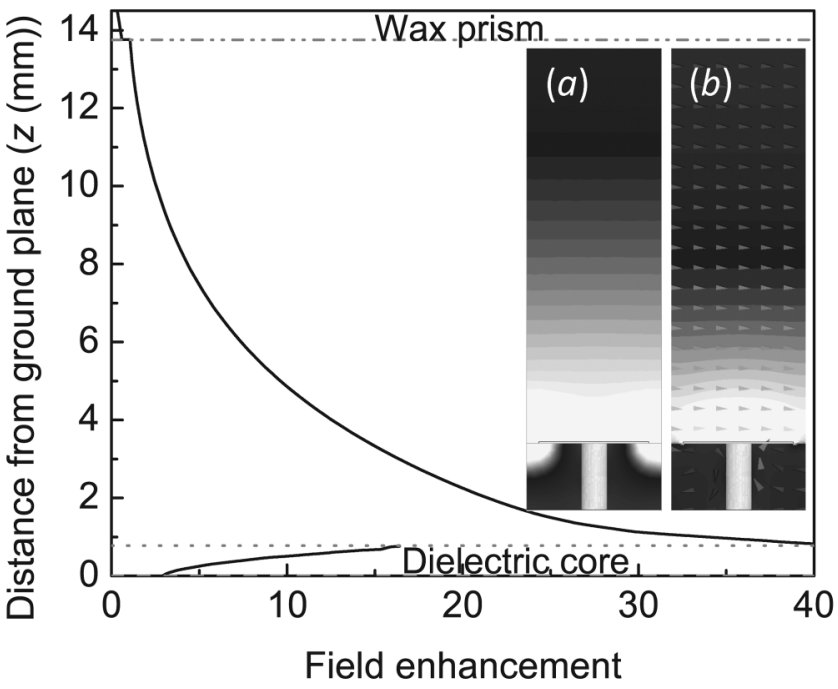

FIG. 3. Field enhancement on resonance for $\theta_{\text {int }}=52^{\circ}$, calculated at a phase corresponding to maximum enhancement, along a line in the $z$ direction (normal to the surface of the array). Here, $z=0 \mathrm{~mm}$ corresponds to the dielectric core-ground plane interface, while $z=0.81 \mathrm{~mm}$ and $z=13.8 \mathrm{~mm}$ corresponds to the patch-tunnel gap and the tunnel gap-wax prism interface, respectively. Inset (a): Time averaged electric field enhancement on resonance for $\theta_{\text {int }}=52.8^{\circ}$, calculated over the $y z$ plane passing through the center of a connecting via. Light regions correspond to enhancements in excess of 40 times the incident field. Inset (b): Poynting vector magnitude (gray scale) and direction (arrows) calculated over the previously mentioned geometry, showing power low at the resonant frequency of the TM surface mode $\theta_{\text {int }}=52^{\circ}$. 
the sample (recall that the overall thickness of the structure represented in Fig. 3(a) is just $0.8 \mathrm{~mm}$ ). The true nature of the decaying fields associated with the surface mode is shown in Fig. 3. Here we see field magnitude calculated as a function of distance along the surface normal to the sample from the ground plane at $z=0 \mathrm{~mm}$ to the wax prism at $z=13.8 \mathrm{~mm}$, evaluated at a phase corresponding to maximum field enhancement. Although the exponential decay of the field above the sample surface is clear $(0.8>$ $z>13 \mathrm{~mm}$ ) it is difficult to evaluate if the fields decaying into the dielectric core of the high impedance surface is indeed exponential due to the small thickness and large refractive index of the core material. However, further finite element modeling (not shown) using a thicker dielectric core and solving for the eigenmodes of the structure (negating the need for an incident medium of wax and the complexity of evaluating the resonance at the optimum coupling condition) indicates an exponential decay of the field in this region also.

The bound surface wave nature of the mode supported here is further demonstrated by Fig. 3(b) (inset). Here, the magnitude (gray scale) and direction (arrows) of the Poynting vector on resonance is shown, once again calculated over a surface in the $y z$ plane through the center of a connecting via for $\theta_{\text {int }}=51.2^{\circ}$. It is clear that strong power flow is confined to the air-metamaterial interface, reducing dramatically away from that interface. Further modeling on resonance (not shown) shows negligible power flow throughout the dielectric region.

In conclusion, we have shown that it is possible to prism couple incident microwave radiation to a bound TM surface mode supported by a "Sievenpiper mushroom" surface which is notably thinner than any other surface used in this regime for this purpose to date. Additionally, we have shown that this bound TM mode is to first order asymptotic to the effective surface plasma frequency defined by the fundamental resonance of the sample. Although EM surface waves have certainly been excited on such a surface previously (hence the use of high impedance surfaces to manipulate the propagation of such waves), to the best of the authors knowledge this is the first experimental study and characterization of a true bound TM surface mode supported by such a surface at microwave wavelengths. Through finite element model- ing we have shown that power flow is strongly confined to the surface of the sample, with exponentially decaying fields into the air. Since this mode is similar to the "spoof plasmon mode" suggested by Pendry et al. [7], but supported by a thin metamaterial surface that is much less than the operating wavelength thick, we suggest that this type of surface has potential to act as a lightweight frequency selective absorber, or with tailored geometrical parameters, to confine, channel or steer power flow across a flat surface.

The authors would like to thank the EPSRC for continued financial support.

[1] K. Uller, dissertation, University Rostock, 1903.

[2] J. Zenneck, Ann. Phys. (Leipzig) 328, 846 (1907).

[3] H. Raether, Surface Plasmons on Smooth and Rough Surfaces and on Gratings (Springer, New York, 1988).

[4] C.C. Cutler, Antennas and Propagation Society International Symposium, AP-S. Digest (IEEE, New York, 1994), Vol. 3, p. 1456.

[5] H. M. Barlow and A. L. Cullen, Proc. Inst. Elect. Eng., London100, 68 (1953); 100, 329 (1953).

[6] R.E. Collin, Field Theory of Guided Waves (IEEE, New York, 1991), 2nd ed.

[7] J. B. Pendry, L. Martín-Moreno, and F. J. Garcia-Vidal, Science 305, 847 (2004).

[8] D. R. Smith, R. C. Vier, W. Padilla, S. C. Nemat-Nasser, and S. Schultz, Appl. Phys. Lett. 75, 1425 (1999).

[9] C.R. Williams, S.R. Andrews, S.A. Maier, A.I. Fernandez-Dominguez, L. Martin-Morenoand, and F. J. Garcia-Vidal, Nat. Photon. 2, 175 (2008).

[10] Y. Chen, Z.Song, Y. Li, M. Hu, Q. Xing, Z. Zhang, L. Chai, and C. Y. Wang, Opt. Express 14, 13021 (2006).

[11] S. A. Maier, S. R. Andrews, L. Martin-Moreno, and F. J. Garcia-Vidal, Phys. Rev. Lett. 97, 176805 (2006).

[12] D. Sievenpiper et al., IEEE Trans. Microwave Theory Tech. 47, 2059 (1999).

[13] www.ansoft.com.

[14] A. Otto, Z. Phys. 216, 398 (1968).

[15] S. Tretyakov, Analytical Modeling in Applied Electromagnetics (Artech House Inc, London, 2003).

[16] N. Engheta and R. W. Ziolkowski, Metamaterials, IEEE Physics and Engineering Explorations (Wiley, New York, 2006). 